\title{
PREVALENCE AND ASSOCIATED RISK FACTORS OF PRE- HYPERTENSION AND HYPERTENSION AMONG ARMY PERSONNEL
}

\author{
Sapana Gurung* \\ Padma Rai*
}

\begin{abstract}
Hypertension (HTN) is most likely a common disease in the world.In every 3 adult ages over 20 years have this disease. As a consequence, Non-communicable diseases (NCDs), such as hypertension and other cardiovascular diseases are becoming increasingly important as causes of mortality and morbidity in all developing countries. The aim of this study is to determine the prevalence of pre-hypertension and hypertension among the Army personnel in Nepal. This was a cross-sectional descriptive study of 315 respondents, who met eligible criteria. All respondents were enumerated by census method using selfinterviewing questionnaire focusing measurement of the blood pressure, weight and height along with the demographic and other socio-economic as well as cultural characteristics. The study shows that overwhelming respondents (77.5\%) are normal. And about 13 percent respondents are seemed to be in the pre-hypertension, followed by stage I HTN (6\%) and stage II HTN (3.5\%). It also shows that there is a significant association between prevalence of hypertension and body mass index of the respondents.
\end{abstract}

Keywords: Prevalence, hypertension, army personnel, No. Six Brigade and Dhading.

\section{INTRODUCTION}

Hypertension (HTN) is most likely a most common disease in the world. Overall 26.4 percent of the world's adult population in 2000 had hypertension and it is expected that by the year 2025, approximately in 3 adult ages over 20 year will have HTN disease (Abdalla et al., 2016). Noncommunicable diseases (NCDs), such as hypertension and other cardiovascular diseases are becoming increasingly important as causes of mortality and morbidity in all developing countries. Communicable diseases continue to have the greatest disease burden but it is estimated that in the next few decades NCDs will outstrip communicable diseases as major cause of death. It clearly indicates that the relative importance of NCDs will increase. This is illustrated by a combination of demographic changes, increasing urbanization and associated changes in levels of risk factors like tobacco smoking, alcohol consumption, obesity and physical inactivity. Hence, in 1994 the prevalence rates of hypertension in the adult population were respectively 8.3 percent. In 1998 these rates increased to 17.6 percent (hypertension) for urban men (Kengne et al., 2007).

Hypertension is a global public health problem with $1 / 4$ adults worldwide estimated to have high blood pressure (BP). The first scientific HTN survey in Nepal was done in 1981 by Mrigendra Samjhana Medical Trust. The prevalence of HTN according to the then used World Health Organization (WHO) criteria (160/95 $\mathrm{mmHg}$ ) in the various parts of the country was as follows: 5.3 percent in Mountains (Jumla), 6 percent in rural Kathmandu (Bhadrabas and Alapot), 8.1 percent in Terai plains (Parsauni), and 9.9 percent in urban Kathmandu (Vaidya et al., 2012).

Hence, that evidence supporting a per-hypertension state has been demonstrated in several studies. Other study showed that, to illustrate the global burden of blood pressure related disease found that most of disease burden caused by high blood pressure in borne by low income, middle income

* Ms. Gurung is principal author, Capt. in Shree Birendra Army Hospital, Chhauni and Ms. Rai is co-author, Lt. Col. in Shree Birendra Army Hospital, Chhauni. 
countries and by people with per-hypertension (Lawes et al., 2008). The substantial burden of suffering associated with HTN , combined with the availability of feasibility and accurate means of detection and clear benefit from treatment have led to worldwide recommendation for HTN screening and management. Hence, available information on the prevalence of HTN and prehypertension is low probably does not reflect the current status of the risk factors for HTN.

\section{METHODOLOGY}

A cross-sectional descriptive study was conducted among army persons from No. Six Brigade, Dhading district. Approvals for conducting the study were obtained from Shree Birendra hospital, No. Six Brigrade and verbal consent was taken from each participant. Total of 315 persons stationed at the Brigrade were enumerated as a census.

\section{Interviewingquestionnaire}

The data was collected using self-administering questionnaire. Each respondent was asked a set of questions if he/she agreed to participate in the study. First, a set of questions related to sociodemographic condition of the respondent was asked, followed by history of chronic disease such as HTN, diabetes and renal problem and a knowledge of hypertension, risk factors of HTN, lifestyle, risk perception and behaviour. In addition, respondents were also asked about life style determinants such as current smoking (smoking up to 1 month before study), alcohol, practices exercise, eating vegetable, fruits and high fatty diet.

Eligible respondents were characterized who agreed the measurements: Arterial blood pressure (ABP), weight (in kg.) and height (In $\mathrm{cm}$.). Blood pressure was measured with Sphygmomanometer and standard protocol. Current ABP classification is based on recent recommendation of the Joint National Committee on Prevention, Detection Evaluation and Treatment of high blood pressure (JNC-7).

Table 1: Classification of ABP among adult population by stages and BMI level

\begin{tabular}{|l|c|c|}
\hline \multirow{2}{*}{ Blood pressure level } & \multicolumn{2}{|c|}{ Range } \\
\cline { 2 - 3 } & $\begin{array}{c}\text { Systolic Blood Pressure (SBP } \\
\mathrm{mmHg})\end{array}$ & $\begin{array}{c}\text { Diastolic Blood Pressure } \\
\text { (DBP mmHg) }\end{array}$ \\
\hline Normal & $<120$ & $<80$ \\
Pre-hypertension & $120-139$ & $80-90$ \\
Stage I HTN & $140-159$ & $90-99$ \\
Stage II HTN & $>160$ & $>100$ \\
Normal weight when BMI & $<25 \mathrm{~kg} / \mathrm{m}^{2}$ & \\
Overweight when BMI & $25-29.99 \mathrm{~kg} / \mathrm{m}^{2}$ & \\
Obesity when BMI & $>30 \mathrm{~kg} / \mathrm{m}^{2}$ respectively. \\
\hline
\end{tabular}

\section{Data collection procedure}

All data was collected by medical personnel who were trained on the methodology of the data surveys, conducted from 1st March, 2015 to 1 April, 2015. Anthropometric measures used standardized methods. Weight and height were measured using scales, and calibrated adult stadiometers. Blood pressure was recorded on the right arm in a seated subject after 10 minute rest, using Sphygmomanometer blood pressure and appropriate cuff sizes. The average of two measurements was taken at two minutes time interval. 


\section{Ethical considerations}

This study was approved by the Nepal Army Institute of Health Science, Research Ethical Committee, and informed consent was obtained from each subject prior to inclusion in the study. Confidentiality of all information obtained from respondents was assured from respondents by confirming the use of data no other than to meet the objective of the study only.

\section{Data management and analysis}

The collected data was checked and organized, if needed coded and recoded. Data entry and cleaning carried out, following with analysis by using SPSS 16 version. Simple cross tabulation was employed for a descriptive analysis and to show inter-relationship between dependent and independent variables. The statistical tools were further applied to show the significant relations and to draw the valid inferences.

\section{Social demographic status}

\section{RESULTS}

Socio-demographic information is taken as an important backdrop on which social, cultural, and health related behaviour of a person is built. Nepal is the least developed country with a much diverse in terms of society, culture, religion, and region. Rural population (83\%) is predominant against urban population (17\%) as National Population and Housing Census (PHC) (CBS, 2012) reported. This study has included types of family, gender, marital status, ethnicity and religion as the social demographic variables.

In types of family, respondents with extended family (a kinship group consisting of a family nucleus and various relatives, as grandparents, usually living in one household and functioning as a larger unit) slightly exceeded the nuclear family (asocialunitcomposedof twoparentsandoneormorechildren) (Table 2).

Table 2: Socio-demographic characteristics of respondents $(n=315)$

\begin{tabular}{|l|r|r|}
\hline Categories & Number & Percent \\
\hline Types of Family & 155 & 49.2 \\
Nuclear & 160 & 50.8 \\
Extended & & \\
Sex & 298 & 94.6 \\
Male & 17 & 5.4 \\
Female & & 65.7 \\
Marital status & 207 & 34.3 \\
Married & 108 & \\
Unmarried & & 28.6 \\
Ethnicity & 90 & 25.1 \\
Chhetri & 79 & 18.1 \\
Others & 57 & 12.4 \\
Brahmin & 39 & 10.2 \\
Indigenous & 32 & 5.7 \\
Newer & 18 & 93.0 \\
Dalit & & 3.2 \\
Religion & 293 & 2.2 \\
Hindu & 10 & 1.6 \\
Buddhist & 7 & \\
Kirant & 5 & \\
Christian \& others & & \\
\hline
\end{tabular}


Respondent with extended family accounted for 51 percent against the nuclear family (49\%). Of the respondents, 95 percent respondents were male army personnel against their female counterparts (5.4\%). And it is due to Nepal Army very recently started to recruit females as the Arm Force in Nepal. About two third respondents were married against 34.3 percent single.

Nepal is a multiethnic, multilingual, and multireligious country. There are more than 123 ethnic and caste groups as PHC, 2011 has reported. According to Dahal (2014), Chhetri is the largest caste group with 16.6 percent, followed by Hill Brahmin (12.2\%), Magar (7.1\%) and Tharu (6.6\%) and so on. In this study of army personnel, respondent of Chhetri caste group accounts for 28.6 percent, followed by Brahmin (18.1\%), indigenous peoples (12.4\%) and so on.

Nepal is also a multi-religious country. However, the religion in South Asia or in Nepal is not as though in European or Western society. In context of Nepal, it is a practice from very beginning a specific caste group has been professing. PHC shows that in overall population, Hindu accounts for 81.3 percent followed by Buddhist (9.0\%), Islam (4.4\%), Kirant (3.0\%), and so on. But respondents with Hindu religion accounts for 93 percent followed by Buddhist (3.2\%), Kirant (2.2\%), Christian (1.3\%) and so on (Table 2).

\section{Hypertension status}

Hypertension is reported as a major cause of morbidity in developing countries. It affects nearly 26 percent of the adult population worldwide. Its prevalence estimates for hypertension may be as much as 1 billion individuals and approximately 7.1 million deaths per year may be attributable to hypertension (WHO, 2002). Studies from India and Bangladesh had shown upward trend in the prevalence of hypertension.

Of the respondents, above 77.5 percent had a normal blood pressure followed by pre-hypertension (13\%), stage-I HTN (6\%) and stage-II HTN (3.5\%) (Table 3). Body Mass Index (BMI) shows that majority of the respondents (69.8\%) had normal followed by overweight (27.6\%) and obesity (2.5\%). Regarding the knowledge about pre-hypertension and hyper tension, most of the respondents (54.3\%) didn't have knowledge about pre-hypertension and hypertension against the respondents of 45.7 percent who had knowledge about them.

Table 3: Distribution of respondents by various issues related to Pre-hypertension and Hypertension $(n=315)$

\begin{tabular}{|l|r|r|}
\hline Categories & Number & Percent \\
\hline Blood Pressure & 244 & 77.5 \\
Normal & 41 & 13.0 \\
pre-hypertension & 19 & 6.0 \\
Stage-I HTN & 11 & 3.5 \\
Stage-II HTN & & \\
Body Mass Index & 220 & 69.8 \\
Normal & 87 & 27.6 \\
Overweight & 8 & 2.5 \\
Obesity & & \\
\hline
\end{tabular}


Table 3: continued

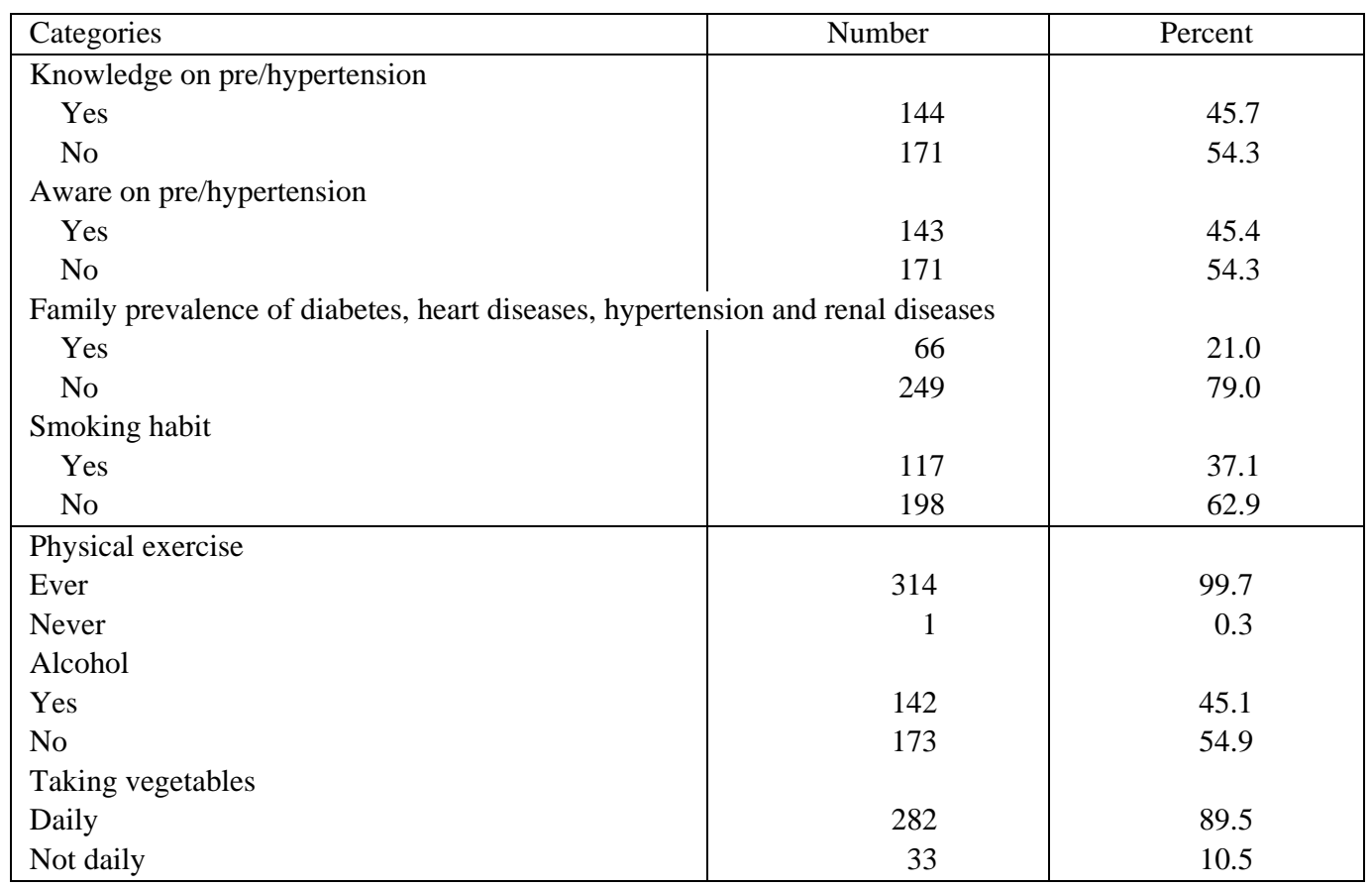

About 54.3 percent respondents were not aware of the pre-hypertension and hypertension and only 45.4 percent were aware about them. About 21 percent of the respondents were from the family with prevalence of diabetes, heart diseases, hypertension and renal diseases. Majority of the respondent's family (79\%) had not such diseases. Only 37.1 percent of the respondents were smokers while majority (62.9\%) were non-smokers.

It is also found that almost all 99.7 percent of the respondents did physical exercises while minimal 0.3 percent didn't have any exercise despite of the military profession. It also shows that majority (54.9\%) of the respondents were non-alcoholic while 45.1 percent were alcoholic. Majority of the respondents (89.5\%) consumed vegetables daily while only 10.5 percent did not consume daily.

\section{Association between dependent and independent variables}

It shows that the $\mathrm{P}$ value is $0.00(<0.05)$ which shows that there is a significant association between prevalence of hypertension and body mass index of the respondents (Table 4).

Table 4: Association between prevalence of hypertension and body mass index of the respondent

\begin{tabular}{|c|c|c|c|c|c|c|}
\hline \multirow{2}{*}{ Body mass index } & \multicolumn{4}{|c|}{ Blood pressure } & \multirow{2}{*}{ Total } & \multirow{2}{*}{$\mathrm{p}$ value } \\
\hline & Normal & Pre-hypertension & $\begin{array}{l}\text { Stage-I } \\
\text { HTN }\end{array}$ & $\begin{array}{c}\text { Stage-II } \\
\text { HTN }\end{array}$ & & \\
\hline Normal & 187 & 22 & 9 & 2 & 220 & \multirow{4}{*}{0.00} \\
\hline Overweight & 56 & 16 & 8 & 7 & 87 & \\
\hline Obesity & 1 & 3 & 2 & 2 & 8 & \\
\hline Total & 244 & 41 & 19 & 11 & 315 & \\
\hline
\end{tabular}


$P$ value is $0.032(<0.05)$ which shows that there is a significant association between prevalence of hypertension and alcohol intake of the respondents (Table 5).

Table 5: Association between prevalence of hypertension and alcohol intake of the respondents

\begin{tabular}{|l|r|r|r|r|r|r|}
\hline \multirow{2}{*}{$\begin{array}{l}\text { Alcohol } \\
\text { consumption }\end{array}$} & \multicolumn{4}{|c|}{ Blood pressure } & \multirow{2}{*}{ Total } & \multirow{2}{*}{ p value } \\
\cline { 2 - 5 } & Normal & Pre-hypertension & $\begin{array}{c}\text { Stage-I } \\
\text { HTN }\end{array}$ & $\begin{array}{c}\text { Stage-II } \\
\text { HTN }\end{array}$ & \\
\hline Yes & 105 & 16 & 14 & 7 & 142 & \multirow{2}{*}{0.032} \\
No & 139 & 25 & 5 & 4 & 173 \\
\hline Total & 244 & 41 & 19 & 11 & 315 & \\
\hline
\end{tabular}

\section{DISCUSSION}

WHO reported that about 27.8 percent of Nepaleseadults aged 25 years and above are estimated tohave Hypertension (Dhital et al., 2013). Among 315 army personnel, 22.2 percent found to be either in pre-hypertension or in hypertension state. It is more likely to see less proportion of army personnel afflicted with HTN than the average Nepali adults, since army personnel are kept more fit in life style and food behaviour than the civil. Nevertheless, the data from the developed world showed a lower incidence rate of hypertension in military population when compared with the general population (Wenzel et al., 2009).

Family and social background also explain the health status in some context. In this study overwhelming respondents (94.6\%) were male, over 50 percent came from extended family, about two third were married, over 93.5 percent Hindu religious, and ethnically diverse groups. Thus potential explanation of background is unlikely, since respondents mostly of the homogenous except the ethnicity.

Nangia et al. (2016) conducted a study on prevalence of known risk factors of cardiovascular diseases (CVD)(like mean body mass index (BMI), systoloic BP and raised cholesterol levels) among armed personnel of 35 years and above age, where the obesity was observed in 3.42 percent. In this study the obesity is observed in 2.5 percent of the total armed personnel interviewed. Majority of the respondents (69.8\%) had normal BMI followed by overweight (27.6\%).

This study shows that about 45 percent respondents were not aware of the pre-hypertension and hypertension. In contrast a study of Mallik et al. (2014) found that around 40 percent of hypertensive knew their status, three-quarter of aware subjects received treatment and only onethird of treated subjects have controlled BP $(<140 / 90 \mathrm{mmHg})$.

This study shows that there is a significant association between prevalence of hypertension and body mass index of the respondents with $\mathrm{p}=0.000(<0.05)$ which is similar to the results of study conducted which also shows the significant association between prevalence of hypertension and $\mathrm{BMI}$ of the respondents being the $\mathrm{p}=0.000$.This study shows that there is a significant association between prevalence of hypertension and alcohol intake of the respondents with $\mathrm{p}=0.032(<0.05)$ 


\section{CONCLUSION}

In spite of most of the respondents are found to be normal blood pressure, there is significant association between the hypertension and body mass index of the respondents and also there is association between hypertension and the alcohol consumption of the respondents. So it can be concluded that lifestyle modifiable factors are related to the hypertension. So, hypertension can be prevented from the lifestyle modification.

\section{REFERENCES}

Abdalla, E., Edrees, A., \& Mohammedahned, A. (2016). Prevalence of hypertension among secondary school students in Umbada area 'Block 14" in Khartoum State-Sudan, Journal of Dental and Medical Sciences, 15(12), 74-78. Retrieved from www.iosrjournals.org.

Central Bureau of Statistics (CBS). (2012). National population and housing census 2011 (National Report), Vol. I. Kathmandu: CBS.

Dahal, D.R. (2014). Social composition of the population: caste/ethnicity and religion in Nepal. Population monograph of Nepal,Vol. II. Kathmandu: CBS, 1-48.

Dhital, S.,\& Karki, A. (2012). Dealing with burden of hypertension in Nepal: Current status, challenges and health system issues. Regional Health Forum, 7(1), 44-52.

Kengne, A. Awah, P.K., Fezeu, L.,\& Mbanya, J. (2007). The burden of high blood pressure and related risk factors in urban Sub-Saharan Africa: Evidences from Douala in Cameroon. African Health Sciences, 7 (1),38-44.

Lawes, C.M., Hoorn, S.V., \& Rodgers, A. (2008). Global burden of bloodpressurerelated disease. The Lancet, 371(9623), 1513-1515.

Mallik, D.,Mukhopadhyay, D.K.,Kumar, P.,\&Sinhababu, A. (2014). Hypertension, prehypertension and normotension among police personnel in a district of West Bengal, India.Assoc Physicians India, 62(11), 6-12.

Nangia, R., Singh, H.,\& Kaur, K. (2016). Prevalence of cardiovascular disease (CVD) risk factors. Medical Journal Armed Forces India, 72(4), 315-319.

Vaidya, A., Pathak, R.P.,\& Pandey, M.R. (2012). Prevalence of hypertension in Nepalese community triples in 25 years: A repeat cross-sectional study in rural Kathmandu. Indian Heart Journal, 64(2), 28-131. Retrieved from doi: 10.1016/S0019-4832(12)60045-5.

Wenzel, D., Souza, J.M.,\& Souza, S.B. (2009). Prevalence of arterial hypertension in young military personnel and associated factors. Rev Saude Publica, 43, 789-795. 
World Health Organization (WHO). (2002).World health report 2002: Reducing risks, promoting healthy life. Geneva, Switzerland: WHO. Retrieved from http://www.who.int/whr/2002. 